\section{Eve de Lamirande Claude Gagnon}

\section{ADRESSE}

E. de Lamirande : docteur ès sciences. C. Gagnon : docteur ès sciences. Laboratoire de recherches en urologie et faculté de médecine, université McGill, hōpital Royal Victoria, 687, avenue des Pins Ouest, Montréal, Québec H3A lAl, Canada.

TIRÉS À PART

\title{
Dérivés actifs de l'oxygène et fonctions du spermatozoïde
}

Les dérivés actifs de l'oxygène ont des effets mixtes sur les spermatozoïdes humains. Le peroxyde d'hydrogène provoque un arrêt total de mobilité dû à un épuisement de l'ATP intracellulaire et à une diminution de phosphorylation des protéines axonémales. En revanche, l'anion superoxyde semble nécessaire à la série de transformations, nommée capacitation, que les spermatozoïdes doivent subir avant de pouvoir féconder l'ovocyte. La nature du dérivé actif de l'oxygène en jeu ainsi que sa concentration et le moment du contact avec les spermatozoïdes sont donc importants pour expliquer les effets observés.

$\mathbf{T}$ ous les organismes qui vivent dans des conditions aérobies font face au paradoxe de l'oxygène: ils ont besoin d'oxygène pour vivre, mais celui-ci peut leur être néfaste ou même mortel s'il est en quantité excessive ou est inadéquatement métabolisé. Dans cet article, nous introduirons d'abord quelques notions de base concernant les dérivés actifs de l'oxygène (DAO) les plus fréquemment rencontrés ; par la suite, nous présenterons les résultats et les concepts les plus récents sur les effets des DAO sur les fonctions des spermatozoïdes.

La réduction complète et séquentielle de l'oxygène $\left(\mathrm{O}_{2}\right)$ pour en arriver à l'eau requiert quatre électrons et implique la formation de DAO (figure 1). La réduction univalente de l'oxygène produit l'anion superoxyde $\left(\mathrm{O}_{2}^{\overline{0}}\right)$ qui, par dismutation spontanée ou enzymatique, engendre le peroxyde d'hydrogène $\left(\mathrm{H}_{2} \mathrm{O}_{2}\right)[1]$. Malgré son faible pouvoir oxydant et sa courte durée de vie $\left(<10^{-3} \mathrm{~s}\right)$ qui le ren- dent relativement peu toxique [1], $\mathrm{O}_{2}^{\bar{a}}$ peut néanmoins inactiver certaines macromolécules comme les RNases et la glycéraldehyde-3-phosphate déshydrogénase [2]. La stabilité et le fort pouvoir oxydatif du $\mathrm{H}_{2} \mathrm{O}_{2}$ rendent ce DAO plus toxique que $\mathrm{O}_{2}^{\circ}$ [1]. L'extrême réactivité du radical hydroxyle $(\bullet \mathrm{OH})$, produit lors de la réduction du $\mathrm{H}_{2} \mathrm{O}_{2}$ par des ions ferreux $\left(\mathrm{Fe}^{+2}\right)$ (réaction de Fenton) ou par la réaction $\mathrm{H}_{2} \mathrm{O}_{2}+\mathrm{O}_{2}^{\bar{亠}}$ en présence d'ions ferriques $\left(\mathrm{Fe}^{3+}\right.$ ) (réaction d'Haber-Weiss) [1], est contrebalancée par sa très courte durée de vie $\left(10^{-9} \mathrm{~s}\right)$. Les DAO sont produits par plusieurs processus cellulaires, dont les chaînes mitochondriales et microsomiales de transport d'électrons, la NADPH oxydase des membranes plasmatiques de plusieurs types cellulaires, certaines réactions métaboliques, etc. [1].

Tous les constituants cellulaires sont des cibles potentielles pour les DAO. L'étendue des modifications oxydatives causées aux protéines, lipides, acides nucléiques et hydrates de car- 
bone dépend de la quantité et de la nature des DAO présents $[1,3]$. Les organismes aérobies ont développé une impressionnante panoplie de mécanismes pour se protéger des effets toxiques des DAO. En première ligne, les petites molécules comme la vitamine $\mathrm{C}$, l'urate, les substances porteuses de groupes sulfhydryles et les enzymes solubles comme la superoxyde dismutase (SOD), la catalase et la glutathion peroxydase piègent les DAO avant qu'ils ne puissent interagir avec leurs cibles cellulaires [ 1 , $3]$. D'autres petites molécules comme l' $\alpha$-tocophérol et la $\beta$-carotène et des enzymes comme la phospholipide hydroperoxyde glutathion peroxydase préviennent ou limitent les dommages secondaires en mettant un terme à la propagation des DAO et de leurs effets. De plus, on reconnaît maintenant que les cellules possèdent des mécanismes de réparation permettant de régénérer les composants endommagés par les DAO [1, $3]$.

Les preuves que les DAO jouent un rôle physiologique dans une variété de processus cellulaires sont récentes. Par exemple, les DAO semblent essentiels à la différenciation cellulaire lors du développement [4]. Un système générateur de $\mathrm{H}_{2} \mathrm{O}_{2}$ module l'iodation des protéines et l'activité du cycle des phosphates de pentose dans la thyroïde du chien [5].

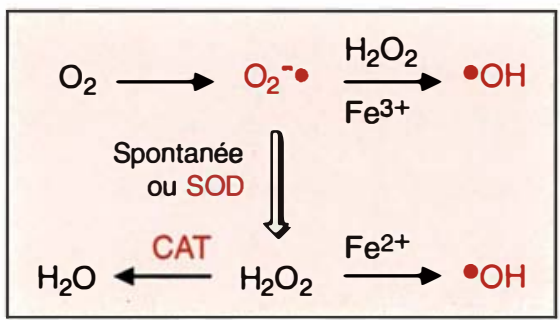

Figure 1. Production des dérivés actifs de l'oxygène. La réduction univalente de l'oxygène $\left(\mathrm{O}_{2}\right)$ produit I'anion superoxyde $\left(\mathrm{O}_{2}^{-}\right)$qui, par dismutation spontanée ou enzymatique (SOD = superoxyde dismutase), engendre le peroxyde d'hydrogène $\left(\mathrm{H}_{2} \mathrm{O}_{2}\right)$. Le radical hydroxyle $(\cdot \mathrm{OH})$ est produit lors de la réduction du $\mathrm{H}_{2} \mathrm{O}_{2}$ en présence d'ions ferreux $\left(\mathrm{Fe}^{+2}\right)$ ou par la réaction $\mathrm{H}_{2} \mathrm{O}_{2}+\mathrm{O}_{2}^{-}$en présence $d^{\prime}$ ions ferriques $\left(\mathrm{Fe}^{+3}\right)$. La catalase (CAT) réduit $\mathrm{H}_{2} \mathrm{O}_{2}$ en $\mathrm{H}_{2} \mathrm{O}$.
Enfin, chez l'oursin de mer, l'œuf utilise une réaction oxydative qui requiert du $\mathrm{H}_{2} \mathrm{O}_{2}$ et la sécrétion d'une peroxydase pour effectuer le pontage entre des groupes tyrosyles et former l'enveloppe de fécondation, prévenant ainsi la polyspermie tout en apportant une protection contre les dangers de l'environnement [6].

\section{Effets toxiques des DAO sur les spermatozoïdes}

Des contenus élevés en acides gras insaturés et relativement faibles en pièges à DAO rendent les spermatozoïdes spécialement sensibles à l'action des oxydants $[7,8]$. Le $\mathrm{H}_{2} \mathrm{O}_{2}$ [913], les peroxydes d'acides gras provenant de la réaction des DAO avec les phospholipides des membranes [14], ainsi que leurs produits de dégradation (hydroxyalkenals, malonaldehyde, etc.) [8, 15] sont aussi toxiques pour les spermatozoïdes. Le niveau de peroxydation des lipides et les dommages cellulaires qui en résultent dépendent non seulement de la nature et de la quantité des DAO présents, mais aussi de facteurs extracellulaires comme la composition ionique du milieu d'incubation, la température et les concentrations d'oxygène, de protéines ou de pièges à $\mathrm{DAO}$ (dans ce milieu par exemple, la taurine et l'hypotaurine) [16].

La génération excessive de DAO par les spermatozoïdes ou par d'autres composants cellulaires du sperme, pourrait diminuer la fertilité chez l'homme $[17,18]$. Une étude récente montre que $25 \%$ des échantillons de sperme de patients infertiles produisent des DAO alors que les échantillons de sperme d'hommes normaux n'en produisent pas [18]. On note également des relations inverses entre le niveau de formation de DAO dans le sperme et le pourcentage de spermatozoïdes mobiles [18], les résultats du test de fusion spermatozoïdes/ovocyte et la fertilité in vivo [17]. Les neutrophiles ainsi que les spermatozoïdes endommagés ou même morphologiquement normaux (mais immobiles ou de biochimie anormale) sont à l'origine des DAO mesurés dans le sperme [18].

Les spermatozoïdes des patients asthénozoospermiques produisent presque deux fois plus de malonaldéhyde (un sous-produit de la peroxydation des lipides) que ceux des hommes fertiles [14]. On observe une corrélation inverse entre la formation de malonaldéhyde et le pourcentage de spermatozoïdes mobiles et une corrélation positive entre la formation de malonaldéhyde et la présence de cellules morphologiquement anormales [19].

On ne connaît pas, actuellement, l'origine de la production accrue de DAO dans les spermatozoïdes des patients infertiles, mais elle pourrait provenir de la diaphorase mitochondriale (une oxydoréductase dépendante du NADH) [20] ou encore d'une hypothétique NADPH oxydase située dans la membrane plasmatique des spermatozoïdes [21].

Pour se protéger des DAO, les spermatozoïdes peuvent compter sur plusieurs mécanismes de défense intraet extracellulaires. Le sperme contient des enzymes telles que la SOD $[8,22]$, la catalase [23] et le système glutathion peroxydase/réductase [10], mais aussi d'autres substances qui peuvent piéger les DAO comme l'albumine [16], le pyruvate [12], la taurine et l'hypotaurine [16], la vitamine C [24], etc. La corrélation directe qui existe entre la durée de mobilité des spermatozoïdes dans le sperme et le niveau de SOD de ces spermatozoïdes confirme l'importance de ces pièges à DAO [8]. De plus, les niveaux élevés de production de DAO que l'on mesure dans le sperme des patients infertiles ne semblent pas dus à une diminution de la capacité de piéger les DAO, mais plutôt à une production accrue de DAO par les cellules du sperme [25].

Les globules blancs, et surtout les neutrophiles, présents dans le sperme peuvent aussi produire des quantités importantes de DAO [26, 27] et donc être potentiellement dangereux pour les spermatozoïdes. Dans un mélange de neutrophiles activés et de spermatozoïdes purifiés par centrifugation sur un gradient de Percoll, on note une baisse de mobilité des spermatozoïdes qui dépend de la durée d'incubation et de la concentration de neutrophiles [27]. L'ajout de catalase et de diméthylsulfoxyde (DMSO) au milieu d'incubation contrecarre totalement les effets néfastes des neutrophiles sur la mobilité des spermatozoïdes [27]. Le plas- 


\section{RÉFÉRENCES}

1. Halliwell B, Gutteridge JMC. Free radicals in biology and medicine, 2nd ed. Oxford: Clarendon Press, 1989.

2. Lavalle F, Michelson AM, Dimitrijevic $\mathbf{L}$. Biological protection by superoxide dismutase. Biochem Biophys Res Commun 1973; 55 : 350-7.

3. Davies KJA, Wiese AG, Sevanian A, Kim EH. Repair systems in oxidative stress. In: Finch CE, Johnson TE, eds. Molecular biology of aging. New York : Wiley-Liss, 1990 : 12341.

4. Allen RG. Oxygen-reactive species and antioxidant responses during development: the metabolic paradox of cellular differentiation. Proc Soc Exp Biol Med 1991; 196 : $117-29$

5. Corvilain B, Van Sande J, Laurent E. The $\mathrm{H}_{2} \mathrm{O}_{2}$-generating system modulates protein iodination and the activity of the pentose phosphate pathway in dog thyroid. Endocrinology 1991; $128: 779-85$

6. Shapiro BM. The control of oxidant stress at fertilization. Science $1991 ; 252$ : 533 6 .

7. Aitken RI, Clarkson JS. Cellular basis of defective sperm function and its association with the genesis of reactive oxygen species by human spermatozoa. / Reprod Fertil 1987 ; $81: 459-69$.

8. Alvarez J, Touchstone J, Blasco L, Storey BT. Spontaneous lipid peroxidation and production of hydrogen peroxide and superoxide in human spermatozoa. Superoxide dismutase as major enzyme protectant against oxygen toxicity. $J$ Androl $1987 ; 8$ : $338-48$.

9. Aitken RJ, Clarkson JS, Fishel S. Generation of reactive oxygen species, lipid peroxidation and human sperm function. Biol Reprod $1989 ; 40: 183-97$.

10. Alvarez JG, Storey BT. Role of glutathione peroxidase in protecting mammalian spermatozoa from loss of motility caused by spontaneous lipid peroxidation. Gamete Res $1989 ; 23$ : 77-90.

11. de Lamirande E, Gagnon C. Reactive oxygen species and human spermatozoa $I$. Effects on the motility of intact spermatozoa and on sperm axonemes. I Androl $1992 ; 13$ : 368-78.

12. de Lamirande E, Gagnon C. Reactive oxygen species and human spermatozoa II. Depletion of adenosine triphosphate plays an important role in the inhibition of sperm motility. J Androl 1992; 13 : 379-86.

13. Aitken RJ, Buckingham D, Harkiss. Use tigate the toxic effects of reactive oxygen species on human spermatozoa. $J$ Reprod Fertil $1993 ; 97: 451-62$

14. Jones R, Mann T, Sherins R. Peroxidative breakdown of phospholipids in human spermatozoa, spermocodal properties of fatty acid peroxides and protective action of seminal plasma. Fertil Steril 1979 ; 31 : 531-7.

15. Selley ML, Lacey MJ, Barlett MR, Copeland CM, Ardlie NG. Content of significant amounts of a cytotoxic end-product of lipid peroxidation in human semen. I Reprod Fertil $1991 ; 92$ : 291-8.

16. Alvarez JG, Storey BT. Taurine, hypotaurine, epinephrine and albumin inhibit lipid peroxidation in rabbit spermatozoa and protect against loss of motility. Biol Reprod $1983 ; 29: 548-55$.

17. Aitken RJ, Irvine DS, Wu FC. Prospective analysis of sperm-oocyte fusion and reactive oxygen species generation as criteria for the diagnosis of infertility. Am J Obstet Gynecol 1991 ; 164 : 542-51.

18. Iwasaki A, Gagnon C. Formation of reactive oxygen species in spermatozoa of infertile patients. Fertil Steril $1992 ; 57$ : 409-16

19. Rao B, Soufir JC, Martin M, David G. Lipid peroxidation in human spermatozoa as related to midpiece abnormalities and motility. Gamete Res $1989 ; 24$ : 127-34.

20. Gavella M, Lipovac V. NADH-dependent oxidoreductase (diaphorase) activity and isozyme pattern of sperm in infertile men. Arch Androl 1992 ; 28 : 135-41.

21. Aitken RJ, Buckingham DW, West KM. Reactive oxygen species and human spermatozoa: analysis of the cellular mechanisms involved in luminol- and lucigenindependent chemiluminescence. $J$ Cell Physiol 1992 ; 151 : 466-77.

22. Nissen HP, Kreysel HW. Superoxide dismutase in human semen. Klin Wochenschr $1983 ; 61: 63-5$.

23. Jeulin C, Soufir JC, Weber P, Laval-Martin D, Calvayrac R. Catalase activity in human spermatozoa and seminal plasma. $G a$ mete Res 1989 ; 24 : 185-96.

24. Dawson EB, Harris WA, Teter MC, Powell LC. Effect of ascorbic acid supplementation on the sperm quality of smokers. Fertil Steril 1992 ; 58 : 1034-9.

25. Zini A, de Lamirande E, Gagnon C. Reactive oxygen species in semen of infertile patients: levels of superoxide dismutaseand catalase-like activities in seminal plasma and spermatozoa. Int J Androl 1993; 16 : 183-8. ma séminal peut aussi protéger les spermatozoïdes des effets toxiques des neutrophiles mais cette protection varie énormément selon l'échantillon de plasma séminal utilisé [27]. On peut donc supposer que la présence de neutrophiles dans le système reproducteur mâle (lors des infections ou de réactions inflammatoires) pourrait causer une diminution de fertilité.

On a d'abord tenté d'expliquer la baisse des fonctions cellulaires des spermatozoïdes due aux DAO par la peroxydation des lipides [14]. Celleci, de même que la formation importante de malonaldéhyde qui en résulte, se produit cependant lorsque les spermatozoïdes sont incubés dans des conditions non physiologiques qui entraînent une perte irréversible de mobilité ainsi qu'une perte de viabilité $[8,10]$.

A concentrations faibles, les DAO agissent au niveau de l'axonème des spermatozoïdes sans affecter la viabilité de ceux-ci $[11,12]$. L'ajout de xanthine et de xanthine oxydase (X $+\mathrm{XO}$, pour produire de l' $\mathrm{O}_{2}^{-}$qui se dismute spontanément en $\mathrm{H}_{2} \mathrm{O}_{2}$ ), à des spermatozoïdes mobiles diminue leur mobilité, mais seulement 3 à 4 heures après le début du traitement, alors que les DAO sont produits puis détruits durant la première heure $[11,12]$. Bien que le traitement avec $\mathrm{X}+\mathrm{XO}$ n'affecte pas le pourcentage de spermatozoïdes mobiles pendant 2 à 3 heures, il provoque, peu de temps après le début de l'incubation, une baisse progressive de la fréquence de battement flagellaire [11]. Celle-ci s'accompagne d'une baisse encore plus rapide du niveau d'ATP intracellulaire $[11,12]$. Ces observations, ainsi que d'autres [11, 12], suggèrent que la baisse d'ATP intracellulaire est une des premières modifications importantes qui déclenche une cascade d'événements conduisant à l'immobilisation des spermatozoïdes [12]. Une des étapes importantes de cette séquence d'événements semble être la baisse de phosphorylation des protéines axonémales [12] (figure 2). Les faibles niveaux d'ATP des spermatozoïdes traités avec des DAO ne suffiraient pas au maintien d'un niveau adéquat de phosphorylation des protéines axonémales [12]. A concentrations beaucoup plus élevées, les DAO en- 
gendrés par $\mathrm{X}+\mathrm{XO}$ provoquent une chute importante de tous les paramètres de mouvement des spermatozoïdes et une production significative de malonaldéhyde [13].

De façon surprenante, dans $50 \%$ des cas, la mobilité des spermatozoïdes immobilisés par de faibles quantités de DAO reprend spontanément 6 à 24 heures après le début du traitement, ce qui suggère que les spermatozoïdes humains possèdent des mécanismes conçus pour réparer les effets des DAO [11]. De plus, le fait que la catalase mais non le DMSO ou la SOD (qui piègent respectivement le radical hydroxyle et $\mathrm{O}_{2}^{\bar{\sigma}}$ ), prévienne la baisse du pourcentage de spermatozoïdes mobiles indique que $\mathrm{H}_{2} \mathrm{O}_{2}$ est le DAO toxique pour les spermatozoïdes humains [11].

\section{Effets bénéfiques des DAO sur les spermatozoïdes}

Les spermatozoïdes de mammifères doivent mener à bien une série de transformations métaboliques et membranaires, globalement nommée capacitation, dans le tractus génital femelle pour que la fécondation soit possible [28]. Ces changements s'associent temporellement à l'acqui-

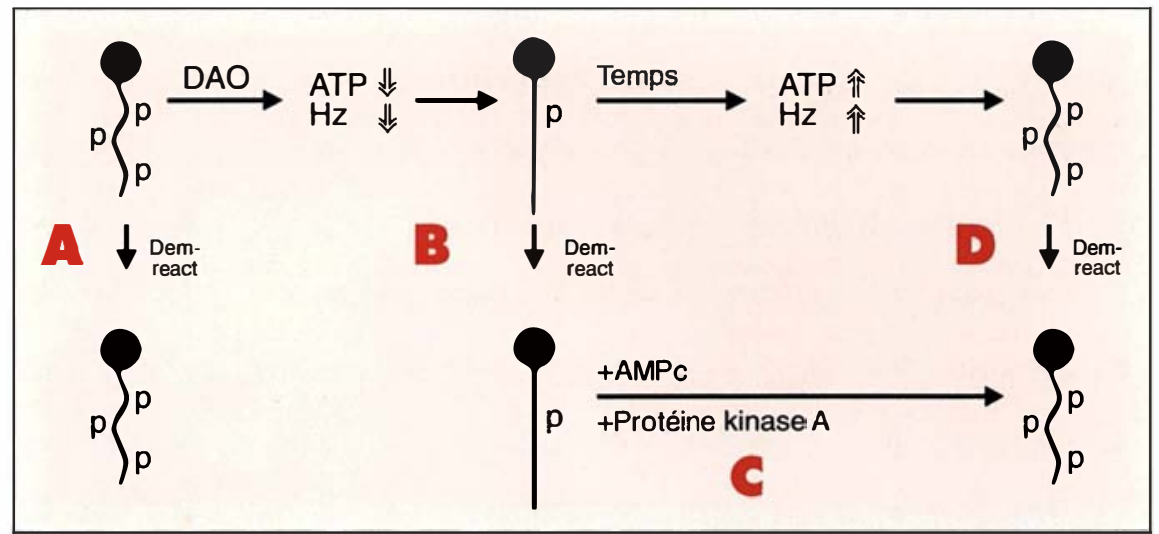

Figure 2. Effets des dérivés actifs de l'oxygène (DAO) sur les flagelles des spermatozoïdes. La portion supérieure de la figure présente les événements qui se produisent dans les spermatozoïdes entiers (en gris) traités ou non avec des DAO alors que la portion inférieure de la figure présente les résultats des tests de démembranation-réactivation (Dem-react) faits sur ces mêmes spermatozoïdes (en noir). A. Le mouvement des spermatozoïdes mobiles, non traités et dilués dans un milieu contenant du Triton X-100 létape de démembranation, Dem) s'arrête immédiatement mais reprend en quelques secondes lors de l'addition de Mg.ATP (étape de réactivation, react). Ce modèle permet d'étudier les mécanismes de contrôle du mouvement des spermatozoïdes ainsi que le mode $d^{\prime}$ action de diverses substances ou traitements qui affectent la mobilité des spermatozoïdes en évitant le problème de perméabilité de la membrane plasmique puisque celle-ci est enlevée par le détergent. B. Les spermatozoides traités par des DAO lqui ont eu un épuisement de leur ATP intracellulaire et une chute de fréquence $(\mathrm{Hz})$ de battement flagellaire jusqu'à arrêt complet de la mobilitél ne peuvent réactiver le mouvement après démembranation dans un milieu contenant Mg.ATP. II y a donc eu des modifications, au niveau de l'axonème des spermatozoïdes, causées par le traitement par des DAO (diminution de la phosphorylation p). C. Les spermatozoïdes immobilisés par le traitement avec des DAO peuvent réactiver le mouvement après démembranation si le milieu contient du Mg.ATP, de l'AMPc et de la protéine kinase A. Cette reprise de mouvement se produit après un délai pendant lequel se refait la phosphorylation axonémale associée au mouvement flagellaire. D. Les spermatozoïdes intacts, qui ont de nouveau une concentration intracellulaire d'ATP compatible avec l'existence du mouvement, réinitient spontanément leur mobilité quelques heures après immobilisation par les DAO et ont une démembranation-réactivation normale. sition d'un type de mobilité très vigoureuse et peu progressive, l'hyperactivation (HA) [28]. Dans les quelques minutes qui suivent l'addition de $\mathrm{X}+\mathrm{XO}$, avant que leur fréquence de battement ne soit diminuée, les spermatozoïdes ont un mouvement très vigoureux. L'ajout de catalase (pour détruire le $\mathrm{H}_{2} \mathrm{O}_{2}$ toxique) à ce milieu permet d'identifier cette mobilité vigoureuse au mouvement hyperactivé [28]. La combinaison $X+$ $\mathrm{XO}+$ catalase (CAT) provoque une augmentation progressive de l'hyperactivation (HA) des spermatozoïdes, le maximum étant atteint 1 à 3 heures plus tard [28]. De plus, la présence de SOD empêche l'augmentation d'HA induite par X + XO + CAT [28]. Ces résultats suggèrent que $\mathrm{O}_{2}^{-}$, produit par la réaction $\mathrm{X}+\mathrm{XO}$, déclenche l'HA des spermatozoïdes [28] et cela malgré sa courte durée de vie (celle-ci est de $1 \mathrm{~ms}$ alors qu'elle chute à 1 ns en présence de SOD [1]).

La forte augmentation d'HA due à $\mathrm{X}$ $+\mathrm{XO}+\mathrm{CAT}$ est très importante au point de vue physiologique car elle s'accompagne d'une augmentation de la capacitation des spermatozoïdes $(\mathrm{X}+\mathrm{XO}+\mathrm{CAT}$ : $23 \pm 1 \%$; milieu seul : $5 \pm 1 \%$ ) [28]. Le besoin $\mathrm{d}^{\prime} \mathrm{O}_{2}^{\overline{-}}$ pour la capacitation des spermatozoïdes humains pourrait être spécifique de cette espèce puisque, chez le hamster, c'est le $\mathrm{H}_{2} \mathrm{O}_{2}$ engendré par la combinaison glucose + glucose oxydase qui permet l'augmentation de la capacitation des spermatozoïdes [29]. Le concept qu'il y ait un DAO spécifique de la capacitation des spermatozoïdes dans chaque espèce est intéressant puisque différents DAO peuvent agir de façon semblable sur des molécules ou groupes fonctionnels (par exemple, groupes sulfhydryles) et causer des effets similaires [1].

L'origine de la production d' $\mathrm{O}_{2}^{-}$ (spermatozoïde et/ou tractus génital femelle) reliée à la capacitation n'est pas encore connue ; on sait, cependant, que l'HA et la capacitation des spermatozoïdes sont inhibés lorsque de la SOD est ajoutée au milieu d'incubation qui normalement provoque l'HA et la capacitation [30, 31] (milieu Ham 5F-10 contenant du sérum de cordon fœtal). Le sérum de cordon fœtal ainsi que d'autres fluides biologiques, le plasma séminal et le 
Figure 3. Corrélation entre la capacitation des spermatozoïdes obtenue avec divers fluides biologiques et le potentiel de ces fluides à piéger I'anion superoxyde. Les divers fluides testés sont : plasma séminal complet (SP), dialysé (SPd), et ultracordon fœtal (FCSu) et de fluide folliculaire (FFu); superoxyde dismutase (SOD) : 0,01 mg/ml. Le coefficient de corrélation $r$ est 0,965 . filtrat (SPu); ultrafiltrat de sérum de

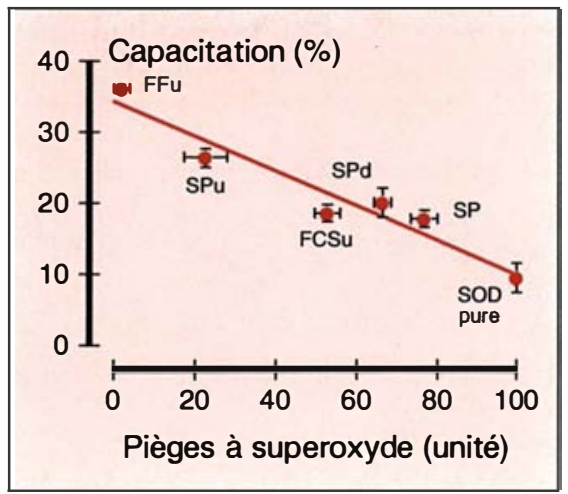

bas que celui que l'on mesure dans le plasma séminal et les spermatozoïdes d'hommes fertiles [33]. Cette HA spontanée dans le sperme affecte probablement le transport des spermatozoïdes le long du tractus génital femelle [34] et/ou entraîne une capacitation prématurée, à un endroit inopportun ; dans les deux cas, le résultat serait une baisse de fertilité. développement de la capacitation [31, 32]. Les niveaux de capacitation obtenus avec les divers fluides biologiques complets, ou leurs fractions, sont très différents, et il existe une relation linéaire inverse entre la capacitation promue par ces fluides et le potentiel de ces fluides à piéger $\mathrm{O}_{2}^{\bar{a}}$ [32] (figure 3). Les fluides biologiques testés contiennent donc tous des promoteurs de la capacitation des spermatozoïdes, et il est possible que ceux-ci agissent tous de la même façon, peut-être en activant directement ou indirectement une NAD(P) $\mathrm{H}$ oxydase (ce qui provoquerait la formation $\mathrm{d}^{\prime} \mathrm{O}_{2}^{\bar{\sigma}}$ ) au niveau de la membrane plasmique des spermatozoïdes. Le potentiel d'un fluide à piéger $\mathrm{O}_{2}^{\overline{-}}$ serait donc un des facteurs les plus importants pour déterminer son action capacitante [32].

L'importance de l'équilibre $\mathrm{O}_{2}^{\bar{a}} /$ pièges à $\mathrm{O}_{2}^{\bar{a}}$ pour le bon fonctionnement des spermatozoïdes est évidente aussi chez les patients infertiles dont les spermogrammes sont "normaux" d'après les critères de l'Organisation mondiale de la santé, mais dans le sperme desquels on retrouve des niveaux d'HA spontanée (16 $\pm 3 \%$ ) bien plus élevés que dans le sperme des volontaires fertiles $(2,6 \pm 0,3 \%)$ [33]. Le potentiel à piéger $\mathrm{O}_{2}^{\bar{\alpha}} \mathrm{du}$ plasma séminal et des spermatozoïdes de ces patients est, respectivement, $37 \%$ et $40 \%$ plus

\section{Conclusion}

Les spermatozoïdes sont très sensibles aux effets des DAO. Ces effets dépendent du DAO impliqué, de sa concentration, ainsi que du moment et de la durée de l'exposition. Même à faibles concentrations, $\mathrm{H}_{2} \mathrm{O}_{2}$ cause une perte réversible de mobilité qui est due surtout à l'épuisement de l'ATP intracellulaire et à l'insuffisance de phosphorylation des protéines axonémales qui en résulte. A forte concentration, $\mathrm{H}_{2} \mathrm{O}_{2}$ provoque une peroxydation des lipides, une perte permanente de fonctions et même la mort cellulaire. Bien que le plasma séminal ait un potentiel énorme à piéger les DAO, il ne peut pas toujours apporter une protection efficace aux spermatozoïdes, surtout quand les DAO sont produits à l'intérieur des cellules. D'un autre côté, $\mathrm{O}_{2}^{\bar{a}}$ exerce un rôle positif sur les spermatozoïdes par son implication dans la promotion et le développement de l'HA et de la capacitation. Les DAO participent donc à la transformation du spermatozoïde le préparant à assumer son rôle fondamental, soit cecette transformation par les DAO ne doit se faire ni trop tôt ni au mauvais endroit lui de féconder l'ovocyte. Cependant,
34. Olds-Clarke $P$, Wivell W. Impaired transted spermatozoa from $+/+$ and concenic tw32/ mice. Biol Reprod $1992 ; 47: 621-8$. 


\section{Summary}

Reactive oxygen species and sperm functions

Reactive oxygen species (ROS) have detrimental or beneficial effects on sperm functions depending on the ROS involved and its concentration, as well as the moment and location of sperm exposure. In humans, the higher level of ROS produced by damaged or deficient spermatozoa is associated with a loss of motility and decreased sperm-oocyte fusion, and can be a cause for idiopathic infertility. Treatment of human spermatozoa with xanthine + xanthine oxidase (X $+\mathrm{XO}$, formation of the superoxide anion which dismutates into hydrogen peroxide) causes a complete loss of motility that is due mostly to depletion of intracellular ATP and a subsequent decreased protein phosphorylation at the axonemal level. Hydrogen peroxide appears to be the ROS responsible for the toxic effects observed. The superoxide anion plays an important role in the transformations, termed capacitation, that ejaculated spermatozoa must undergo before they fertilize oocytes. The combination $\mathrm{X}+\mathrm{XO}$ in the presence of catalase induces higher levels of capacitation than a known biological inducer of this process (fetal cord serum). Superoxide dismutase prevents the capacitation observed with $\mathrm{X}+\mathrm{XO}+$ catalase as well as that observed with fetal cord serum, seminal plasma, and follicular fluid. Furthermore, the superoxide scavenging capacity of the biological fluids mentioned appears to be one of the determining factors influencing their ability to induce sperm capacitation.

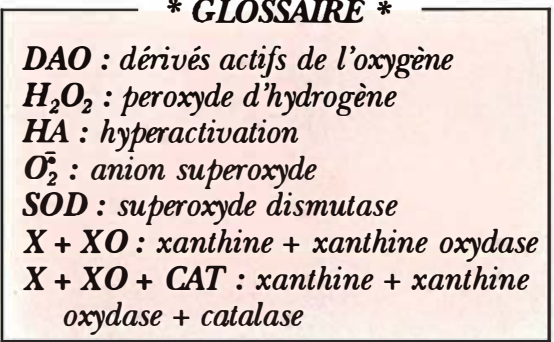

